\title{
A Very Compact and Sharp Roll-Off Low-Pass Filter with Four Transmission Zeros
}

\author{
Yang Xiao and Lin Li \\ Department of Electronic Engineering, Zhejiang Sci-Tech University, Hangzhou, Zhejiang 310018, China
}

Correspondence should be addressed to Lin Li; lilin_door@hotmail.com

Received 30 April 2015; Accepted 30 July 2015

Academic Editor: Flavio Canavero

Copyright ( 2015 Y. Xiao and L. Li. This is an open access article distributed under the Creative Commons Attribution License, which permits unrestricted use, distribution, and reproduction in any medium, provided the original work is properly cited.

A novel structure with sharp roll-off, wide stopband, and very compact size is presented in this paper. By combining a capacitorembedded transmission line ring and two shunt open stubs, this structure exhibits a high-performance three-pole low-pass filter (LPF) response with four generated transmission zeros. With the help of these four transmission zeros, the proposed LPF achieves improved roll-off rate, extended stopband, and significantly very compact size. To verify the feasibility of the proposed structure, a prototype LPF having the cut-off frequency at $0.63 \mathrm{GHz}$ is designed, fabricated, and measured as an illustrative example. Final result shows that a roll-off rate of $109.3 \mathrm{~dB} / \mathrm{GHz}$ along with a relative stopband bandwidth of $114.6 \%$ can be obtained. Moreover, the filter dimensions are as small as $15.7 \mathrm{~mm} \times 26.9 \mathrm{~mm}$, that is, $0.004 \lambda_{g}{ }^{2}$, where $\lambda_{g}$ is the guided wavelength at the cut-off frequency. The filter structure is simple and easy to fabricate as well.

\section{Introduction}

High-performance low-pass filters (LPFs) with the compact size are highly desirable to remove the unwanted highfrequency harmonics and intermodulations in various wireless communication systems. Conventional transmission line LPFs, such as open-circuited stub and stepped-impedance LPFs, often suffer from gradual cut-off attenuation skirt, narrow stopband bandwidth, and large circuit size [1]. For achieving the requirement of high performance, the usual approach is to raise the order of stepped-impedance LPFs to a great extent. Unfortunately, both the circuit size and in-band insertion loss increase considerably.

Recently, many studies in defected ground structure (DGS) have been made to obtain LPF with sharp rolloff rate, wide stopband characteristic, and compact size [2, 3]. However, the design procedure of DGS has fabrication difficulties and DGS itself will cause radiation. Except for DGS, another method to realize compact LPF is to introduce transmission zeros in the filter's transfer function. Generating transmission zero points and properly locating them can develop sharp response from passband to stopband and deep rejection outside the passband. Thus, a low-order LPF with the help of extra transmission zero points can meet the stop band requirements that are usually achieved by high-order filters. In [4], the stepped-impedance hairpin resonator is adapted to design wideband LPF. However, the roll-off skirt of this LPF is very gradual as only one transmission zero is excited in the stopband, which is very far away from the cutoff frequency. And, in $[5,6]$, two transmission zeros are produced to improve the stopband behavior by introducing the CSRR (complementary split ring resonators) and interdigital DGS, respectively. Nevertheless, the stopband characteristics remain to be enhanced due to finite transmission zeros. Therefore, in order to excite multiple transmission zeros to broaden the stopband and sharpen the roll-off skirt, multisection structures have been engineered in [7-9], for instance, in [7], a cascaded unit composed of two asymmetrical hairpin units as well as a connecting line is employed to obtain a very wide stopband LPF with five proposed transmission zeros; additionally in [8, 9], the cascades of various TFSs (transversal filtering sections) and several CPW (coplanar-waveguide) tapered sections are exploited to obtain an extraordinary sharp-rejection, ultralarge attenuated band LPF with multiple transmission zeros. Despite so many attractive features, these multisection LPFs [7-9] generally have large circuit size, which greatly hinder their further developments and applications. Owing 
to the gradual roll-off skirt, complex fabrication, and large circuit size, these structures fail to meet the increasing higher demand of high-performance LPFs with the compact size.

In this paper, a novel structure composed of a capacitorembedded ring as well as two symmetrical open stubs connected at the two bilateral tapped points is proposed to solve the problem above. Detailed analysis based on matrices transformation reveals that the proposed structure exhibits three-pole low-pass filtering response with four adjustable transmission zeros. By properly adjusting and locating the four transmission zeros, not only sharp and wide rejection but also size reduction can be addressed simultaneously. The design, simulation, and measurement of the exemplary filter successfully verify the feasibility of the proposed structure to realize high-performance LPF with very compact size.

\section{Theoretical Analysis}

Figure 1 demonstrates the basic configuration of the proposed low-pass filter. As shown in the figure, the proposed lowpass filter consists of two parallel sections, that is, two symmetrical open-circuited transmission lines in Figure 2(a) and a capacitor-embedded transmission line ring in Figure 2(b). To analyze this structure easily, we assume that the whole transmission line model is lossless and the effect of the feeding lines and all discontinuities is negligible.

$$
\left[Y_{a}\right]=\left[\begin{array}{c}
\frac{1}{j Z_{1} \tan \theta_{1}}+\frac{\cos ^{2} \theta_{2}+Y_{2} \sin \theta_{2} \cos \theta_{2} / w c-\sin ^{2} \theta_{2}}{2 j Z_{2} \sin \theta_{2} \cos \theta_{2}+\cos ^{2} \theta_{2} / j w c} \\
j Y_{2} \csc \theta_{2}-\frac{1}{2 j Y_{2} \sin \theta_{2} \cos \theta_{2}+j Y_{2} \sin ^{2} \theta_{2} / w c}
\end{array}\right.
$$

where $Z_{1}$ and $Z_{2}$ are the upper and lower transmission line's characteristic impedance, respectively; $\theta_{1}$ and $\theta_{2}$ are the upper and lower transmission line's electrical length, respectively; $w$ is the angular frequency $(w=2 \pi f) ; c$ is capacitance of the embedded capacitor.

As to the network shown in Figure 2(b), the $Y$-matrix can be given as

$$
\begin{aligned}
& {\left[Y^{T}\right]} \\
& =\left[\begin{array}{c}
\frac{1}{j Z_{1} \tan \theta_{1}}+\frac{\cos ^{2} \theta_{2}+Y_{2} \sin \theta_{2} \cos \theta_{2} / w C-\sin ^{2} \theta_{2}}{2 j Z_{2} \sin \theta_{2} \cos \theta_{2}+\cos ^{2} \theta_{2} / j \omega C}+\frac{j \tan \theta_{3}}{Z_{3}} \\
j Y_{2} \csc \theta_{2}-\frac{1}{2 j Y_{2} \sin \theta_{2} \cos \theta_{2}+j Y_{2} \sin ^{2} \theta_{2} / w C}
\end{array}\right.
\end{aligned}
$$$$
\left.\begin{array}{c}
j Y_{2} \csc \theta_{2}-\frac{1}{2 j Y_{2} \sin \theta_{2} \cos \theta_{2}+j Y_{2} \sin ^{2} \theta_{2} / w C} \\
\frac{1}{j Z_{1} \tan \theta_{1}}+\frac{\cos ^{2} \theta_{2}+Y_{2} \sin \theta_{2} \cos \theta_{2} / w C-\sin ^{2} \theta_{2}}{2 j Z_{2} \sin \theta_{2} \cos \theta_{2}+\cos ^{2} \theta_{2} / j w C}+\frac{j \tan \theta_{3}}{Z_{3}}
\end{array}\right] .
$$

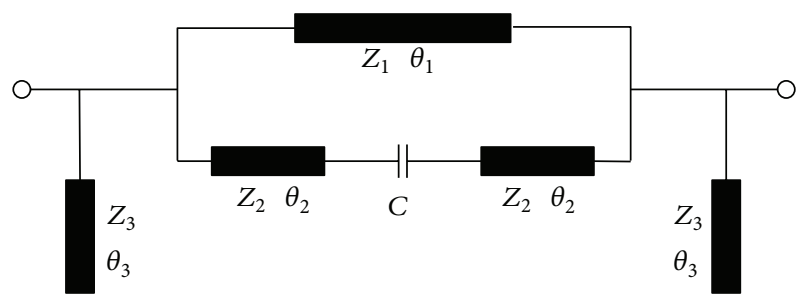

FIGURE 1: The proposed LPF structure.

Based on the assumptions above, the total $Y$-matrix of the structure can be expressed as

$$
[Y]=\left[Y_{a}\right]+\left[Y_{b}\right]
$$

where $\left[Y_{a}\right]$ and $\left[Y_{b}\right]$ are the admittance matrices of networks in Figures 2(a) and 2(b), respectively.

As for the network in Figure 2(a), the overall $Y$-matrix can be derived as

$$
\left.\begin{array}{c}
j Y_{2} \csc \theta_{2}-\frac{1}{2 j Y_{2} \sin \theta_{2} \cos \theta_{2}+j Y_{2} \sin ^{2} \theta_{2} / w c} \\
\frac{1}{j Z_{1} \tan \theta_{1}}+\frac{\cos ^{2} \theta_{2}+Y_{2} \sin \theta_{2} \cos \theta_{2} / w c-\sin ^{2} \theta_{2}}{2 j Z_{2} \sin \theta_{2} \cos \theta_{2}+\cos ^{2} \theta_{2} / j w c}
\end{array}\right],
$$

$$
\left[Y_{b}\right]=\left[\begin{array}{cc}
j \tan \frac{\theta_{3}}{Z_{3}} & 0 \\
0 & j \tan \frac{\theta_{3}}{Z_{3}}
\end{array}\right]
$$

here, $Z_{3}$ and $\theta_{3}$ represent the characteristic impedance and electrical length of the two symmetrical open-circuited transmission lines.

Then the total $Y$-matrix in Figure 1 can be given by

capacitance of the single upper transmission line, respectively. $C_{g}$ and $C_{p}$ represent the equivalent capacitance of the embedded capacitor and two lower transmission lines,
Analyzing the proposed structure in Figure 1, we introduce an equivalent $\pi$-circuit model displayed in Figure 3, where $L_{S}$ and $C_{S}$ are the equivalent inductance and stray 


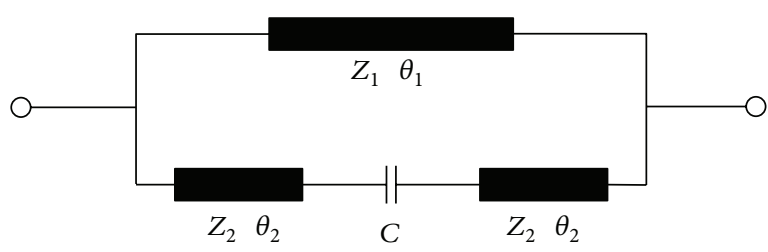

(a)

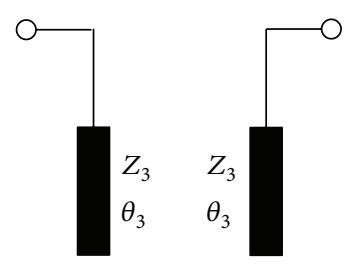

(b)

Figure 2: (a) Capacitor-embedded transmission line ring. (b) Two symmetrical open-circuited transmission lines.

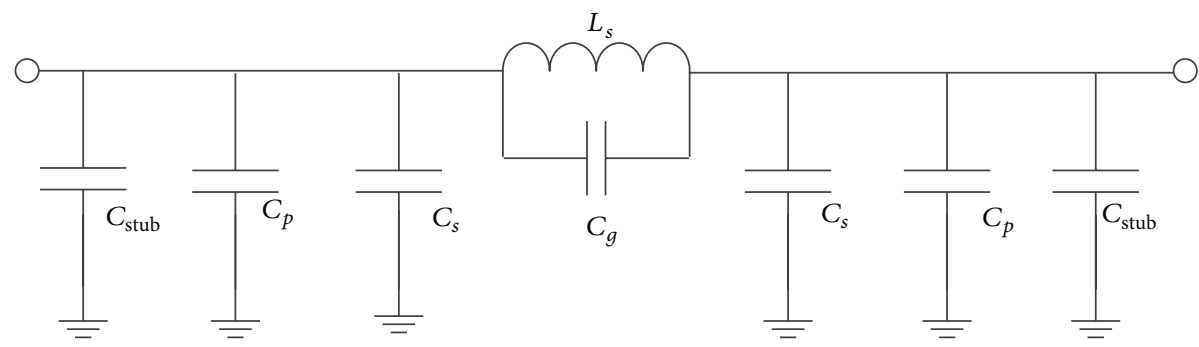

FIGURE 3: Equivalent $\pi$-circuit model of the proposed structure.

respectively. Finally, $C_{\text {stub }}$ is found as the equivalent capacitance of the two open-circuited transmission lines jointed at two ends. Thus the overall structure can be equivalent as a $\pi$ circuit model. Furthermore, the parameters of the proposed equivalent $\pi$-circuit can be described as

$$
\begin{aligned}
L_{s} & =\frac{Z_{1} \sin \theta_{1}}{w}(H), \\
C_{s} & =\frac{1-\cos \theta_{1}}{w Z_{1} \sin \theta_{1}}(F), \\
C_{g} & =\frac{C}{\cos ^{2} \theta_{2}-2 w C \sin \theta_{2} \cos \theta_{2} / Y_{2}}(F), \\
C_{p} & =\frac{2 c \sin ^{2} \theta_{2}+\sin \theta_{2} \cos \theta_{2} Y_{2} / w}{\cos ^{2} \theta_{2}-2 w c \sin \theta_{2} \cos \theta_{2} / Y_{2}}(F), \\
C_{\text {stub }} & =\frac{Z_{3} \tan \theta_{3}}{w}(F) .
\end{aligned}
$$

Obviously, the proposed structure behaves quasielliptic low-pass filtering characteristics. By using the classic method at the cut-off frequency, the prototype element values in Figure 3 can be synthesized and then the physical dimensions of the proposed structure can also be obtained. To confirm the validity of the presented equivalent model and corresponding synthesis method, a low-pass filter using the proposed structure is designed to provide a $0.1 \mathrm{~dB}$ equalripple Chebyshev response. The optimized parameters of the proposed structure are $Z_{1}=105 \Omega, Z_{2}=90 \Omega$, and $Z_{3}=$ $48.5 \Omega ; \theta_{1}=120^{\circ}, \theta_{2}=8^{\circ}$ at $2 \mathrm{GHz}$, and $\theta_{3}=90^{\circ}$ at $1 \mathrm{GHz}$; $C=0.75 \mathrm{pF}$. Both the ideal response and the response of the synthesized circuit are shown in Figure 4. And to compare the proposed structure and the conventional LPF, the response

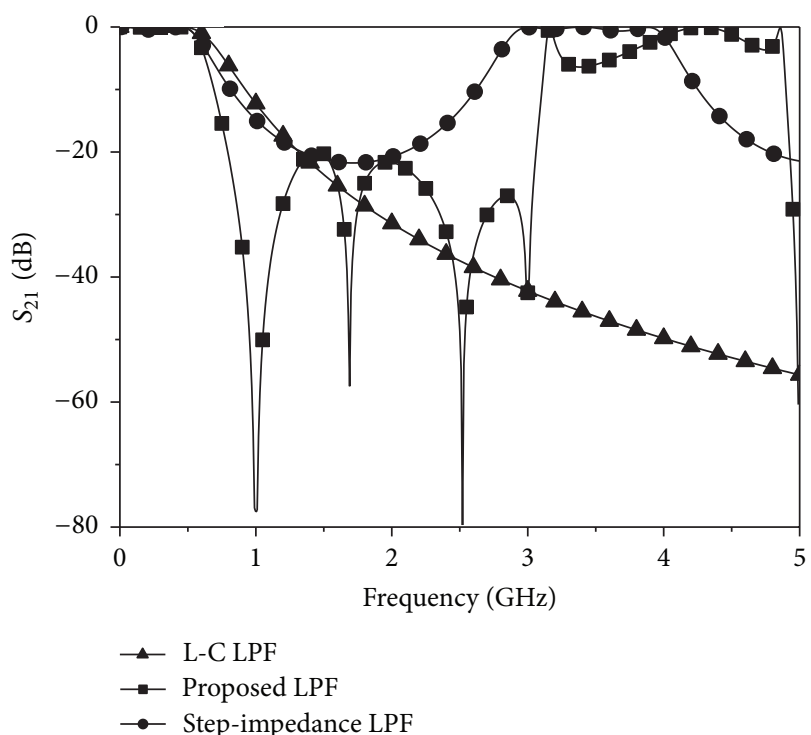

FIGURE 4: Simulated responses of the L-C LPF, proposed LPF, and the step-impedance LPF.

of the conventional stepped-impedance LPF is also displayed in Figure 4. Good agreement can be observed, indicating the feasibility of the proposed structure and the synthesis method.

As evidently illustrated in Figure 4, multiple transmission zeros appear on the response of the proposed structure. With the help of the four transmission zeros, the proposed structure exhibits much higher selectivity and much wider stopband than the conventional stepped-impedance LPF.

The transmission zeros of the proposed structure are located at the frequency where $S_{21}=0$. From the relationship 
between the admittance matrices and scattering matrices, the scattering matrices of the total network can be given by

$$
S_{21}=\frac{-2 Y_{21} Y_{0}}{\left(Y_{11}+Y_{0}\right)\left(Y_{22}+Y_{0}\right)-Y_{12} Y_{21}},
$$

where $Y_{0}$ is the characteristic admittance of the input port and the output port.

Then by setting $Y_{21}=0$ or $Y_{11}=\infty, S_{21}=0$ can be obtained and the frequency of the finite transmission zeros should satisfy either of the following equations:

$$
\begin{aligned}
& \frac{\csc \theta_{1}}{Z_{1}}=2 \pi f C_{g}, \\
& \tan \theta_{3}=\infty .
\end{aligned}
$$

Apparently, the transmission zeros come from the counteraction of the two signal paths in Figure 2(a) and the series resonance of the bilateral two open stubs in Figure 2(b). Besides, the two transmission zero generation mechanisms are mutually independent, which provide great flexibility for filter analysis and design.

Firstly, the structure in Figure 2(a) has a similar topology to the filter in [10]. In fact, the structure in Figure 2(a) will be deformed to the filter in [10] when $\theta_{2}=0$. As illustrated in [10], a larger parallel capacitor creates larger transmission zero separations. And it can be deduced from (5c) that the introduction of the bilateral transmission line $(Z=90 \Omega$, $\theta=8^{\circ}$ at $2 \mathrm{GHz}$ ) will largely magnify the capacitance of $C$. As a result, the proposed structure can generate two transmission zeros with much wider zero separation and thus ensure a wider rejection band. Besides, the introduction of a larger parallel capacitor can not only lower the filter's cutoff frequency but also enhance the selectivity considerably. Furthermore, a larger parallel capacitor indicates a more remarkable size reduction of the upper line's electrical length, meaning that the whole circuit can be further minimized.

As for the structure in Figure 2(b), based on the two generated transmission zeros derived from (7), another two transmission zeros $f_{z 3}$ and $f_{z 4}$ can be obtained when $\theta_{3}=$ $\pi / 2$ and $\theta_{3}=3 \pi / 2$, which makes $f_{z 4}=3 f_{z 3}=3 \mathrm{GHz}$; hence the overall frequency response with four transmission zeros is realized. Besides, the locations of $f_{z 3}$ and $f_{z 4}$ can be shifted flexibly and easily by altering the electrical length of the two symmetrical open-circuited lines depending on the designed goal. Evidently, when the locations of $f_{z 1}$ and $f_{z 2}$ have been predetermined, the locations of $f_{z 3}$ and $f_{z 4}$ have a significant influence on the filter's overall frequency responses. Different transmission zero's locations will lead to different frequency responses. Then select an appropriate $\theta_{3}$ and let the $f_{z 3}$ locate before $f_{z 1}$ while the $f_{z 4}$ locate behind the $f_{z 2}$; to do so, the roll-off rate will be significantly upgraded as the result of the effect of $f_{z 3}$; meanwhile, the stopband bandwidth will extend to the upper side and create wider stopband owing to the impact of $f_{z 4}$. Therefore, as obviously depicted in Figure 5, the proposed low-pass filter with two symmetrical opencircuited lines ensures a wider stopband, sharper roll-off rate, and deeper rejection level. Apart from these interesting characteristics, the open-circuited lines hardly brings about

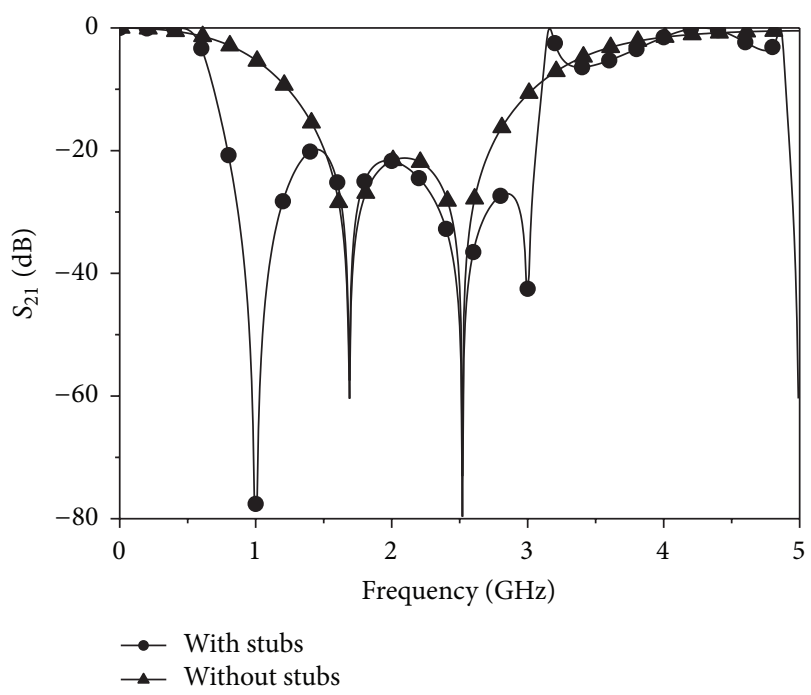

FIGURE 5: The simulated frequency response with and without the stubs.

influence to passband frequency response meaning that it is convenient for further design and adjustment. Furthermore, the introduced open-circuited transmission lines can be folded easily so as to contribute to the miniaturization of the whole circuit, thereby achieving a balance between the excellent performance and the compact size.

\section{Fabrication and Measurements}

Based on the above-discussed attractive features, a prototype LPF having $f c$ of $0.63 \mathrm{GHz}$ and a wide $20 \mathrm{~dB}$ stopband extended up to $5 f c$ is implemented on a substrate with thickness of $0.5 \mathrm{~mm}$ and relative dielectric constant of 2.65 and loss tangent of 0.02 . The physical dimensions along with the photograph of the proposed LPF are demonstrated in Figures 6(a) and 6(b). The parameters of the proposed structure are arranged as follows: $w_{1}=0.33 \mathrm{~mm}, l_{1}=$ $14.15 \mathrm{~mm}, w_{2}=0.47 \mathrm{~mm}, l_{2}=2.32 \mathrm{~mm}, w_{3}=1.3 \mathrm{~mm}$, $l_{3}=11.92 \mathrm{~mm}, l_{4}=13.3 \mathrm{~mm}, w_{50}=1.34 \mathrm{~mm}, l_{50}=$ $25.18 \mathrm{~mm}$, and $C=0.75 \mathrm{pF}$ which are achieved by using the MURATA patch capacitors with the size of $1.6 \mathrm{~mm} \times 0.8 \mathrm{~mm}$. Meandered lines are adapted to achieve a very compact area. The Ansoft software High Frequency Structure Simulator (HFSS) is used for the microstrip line simulations while the Agilent $8510 \mathrm{C}$ vector network analyzer is applied for the corresponding measurements.

Figure 7 shows an excellent agreement between the measured and simulated frequency responses; the measured $3 \mathrm{~dB}$ cut-off frequency is $0.63 \mathrm{GHz}$ and the passband insertion loss including the SMA connector loss is within $0.5 \mathrm{~dB}$ up to $0.54 \mathrm{GHz}$, while the return loss is better than $12 \mathrm{~dB}$ up to $0.31 \mathrm{GHz}$. The measured four transmission zeros are located at $1.1 \mathrm{GHz}$ (with a suppression of $59.15 \mathrm{~dB}$ ), $1.76 \mathrm{GHz}$ (with a suppression of $41.45 \mathrm{~dB}), 2.75 \mathrm{GHz}$ (with a suppression of $79.42 \mathrm{~dB}$ ), and $3.1 \mathrm{GHz}$ (with a suppression of $45.6 \mathrm{~dB}$ ), respectively. With the aid of these transmission zeros, the 
TABLE 1: Comparison with the reported LPFs.

\begin{tabular}{lccccc}
\hline References & RO $(\mathrm{dB} / \mathrm{GHz})$ & $f_{\mathcal{c}}(\mathrm{GHz})$ & Up to $(f c)$ & $\begin{array}{c}\text { Stopband } \\
\text { Demon. FBW }(\%) \text { Rej. }(\mathrm{dB})\end{array}$ & $\mathrm{Ckt}$ size $\left(\lambda_{g}{ }^{2}\right)$ \\
\hline$[3]$ & 34 & 2.4 & 3.4 & 95,20 & 0.049 \\
{$[4]$} & 68 & 2.0 & 5 & $120,33.5$ & Large \\
{$[5]$} & 130 & 1.8 & 3.4 & Unknown & 0.02 \\
{$[7]$} & 37 & 2.5 & 4.8 & 115,20 & $135,21.5$ \\
{$[8]$} & 270 & 1.0 & 5.3 & 140,20 & 0.022 \\
{$[11]$} & 95 & 0.5 & 9 & $\mathbf{1 1 4 , 2 0}$ & Large \\
This work & $\mathbf{1 0 9 . 3}$ & $\mathbf{0 . 6 3}$ & $\mathbf{5}$ & & $\mathbf{0 . 0 0 4}$ \\
\hline
\end{tabular}

RO: roll-off; Demon.: demonstrated; FBW: fractional bandwidth; Rej.: rejection level; Ckt.: circuit.

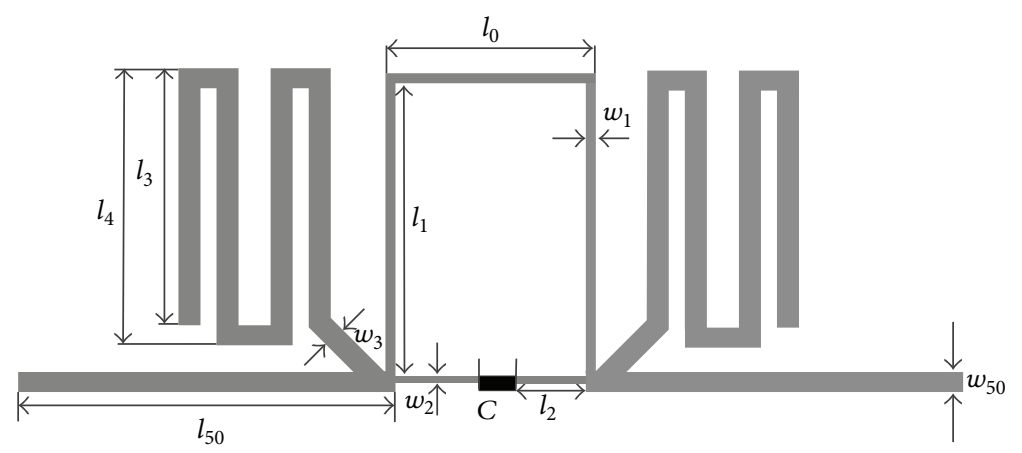

(a)

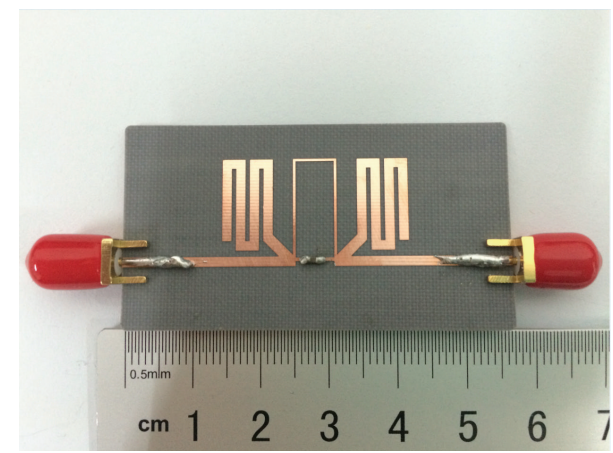

(b)

Figure 6: (a) The layout and physical dimensions of the proposed LPF. (b) The photograph of the proposed LPF.

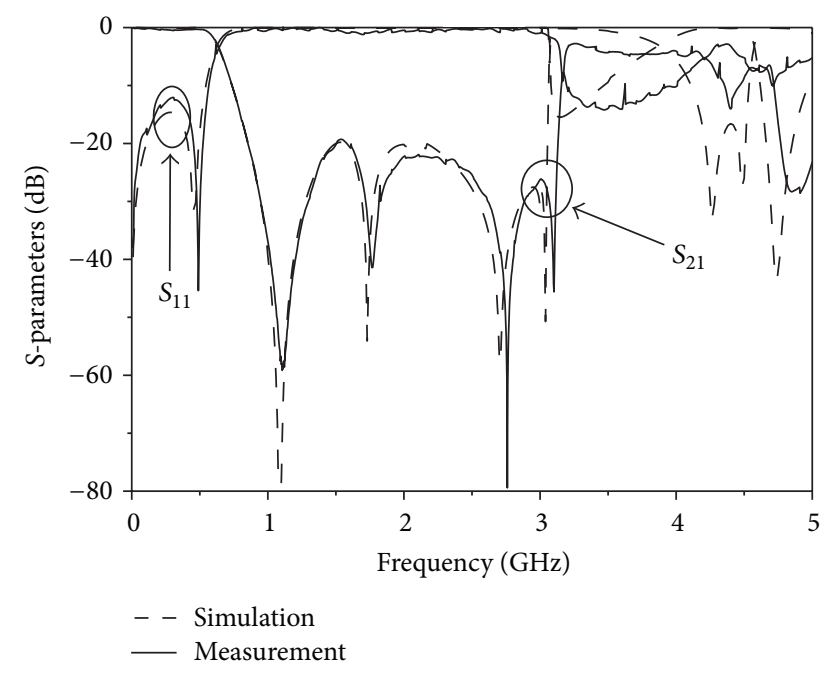

FIGURE 7: Simulated and measured frequency responses of the fabricated LPF.

measured $20 \mathrm{~dB}$ rejection stopband extends from $0.85 \mathrm{GHz}$ to $3.13 \mathrm{GHz}$, forming a very wide attenuation band with the $20 \mathrm{~dB}$ rejection bandwidth up to $5.04 f c$. Moreover, these deep rejection transmission zeros also create a sharp transition for the filter; thus the proposed LPF exhibits an incredible rolloff rate of $109.3 \mathrm{~dB} / \mathrm{GHz}$ (measured attenuations being $3 \mathrm{~dB}$ and $50 \mathrm{~dB}$ at $0.63 \mathrm{GHz}$ and $1.06 \mathrm{GHz}$, resp.). In addition, the central circuit (without the consideration of the $50 \Omega$ feed line) occupies a really compact size of $26.9 \mathrm{~mm} \times 15.7 \mathrm{~mm}$, corresponding to $0.004 \lambda_{g}{ }^{2}\left(0.083 \lambda_{g} \times 0.049 \lambda_{g}\right)$, where $\lambda_{g}$ is the guided wavelength of a $50 \Omega$ transmission line at the cutoff frequency $0.63 \mathrm{GHz}$.

A comparison of the proposed LPF with other reported compact sharp roll-off LPFs is provided in Table 1. Compared with the LPFs in the table, the proposed low-pass filter behaves a wide stopband and rather steep roll-off rate. Furthermore, the size of the proposed LPF is the smallest among the LPFs in Table 1, indicating that it is a good candidate to satisfy the requirement of miniaturization.

\section{Conclusions}

The design of a compact sharp roll-off low-pass filter based on the novel structure is presented in this paper. It is analyzed in theory that the proposed structure can produce four adjustable transmission zeros by changing the parameters of the circuit to obtain the wanted LPF. Applying the analysis method, a prototype LPF is designed, fabricated, and tested. Measured results agree well with the theoretical predictions and demonstrate its incredible features: sharp roll-off rate, wide stopband, and very compact size. With all these charming characteristics, the proposed LPF is very applicable in the modern communication system. 


\section{Conflict of Interests}

The authors declare that there is no conflict of interests regarding the publication of this paper.

\section{Acknowledgments}

This work was supported by the program for Zhejiang leading team of science and technology innovation (2011R50004), the National Natural Science Foundation of China under Grant 61101052 and 521 talent project of Zhejiang Sci-Tech University.

\section{References}

[1] J.-S. G. Hong and M. J. Lancaster, Microstrip Filter for RF/Microwave Applications, Wiley, New York, NY, USA, 2001.

[2] R. Sharma, T. Chakravarty, S. Bhooshan, and A. B. Bhattacharyya, "Design of a novel 3 DB microstrip backward wave coupler using defected ground structure," Progress in Electromagnetics Research, vol. 65, pp. 261-273, 2006.

[3] Y. Jinping and W. Wen, "Compact elliptic-function low-pass filter using defected ground structure," IEEE Microwave and Wireless Components Letters, vol. 18, no. 9, pp. 578-580, 2008.

[4] L.-H. Hsieh and K. Chang, "Compact elliptic-function low-pass filters using microstrip stepped-impedance hairpin resonators," IEEE Transactions on Microwave Theory and Techniques, vol. 51, no. 1, pp. 193-199, 2003.

[5] M. K. Mandal, P. Mondal, S. Sanyal, and A. Chakrabarty, "Low insertion-loss, sharp-rejection and compact microstrip lowpass filters," IEEE Microwave and Wireless Components Letters, vol. 16, no. 11, pp. 600-602, 2006.

[6] A. Balalem, A. R. Ali, J. Machac, and A. Omar, "Quasi-elliptic microstrip low-pass filters using an interdigital DGS slot," IEEE Microwave and Wireless Components Letters, vol. 17, no. 8, pp. 586-588, 2007.

[7] S. Luo, L. Zhu, and S. Sun, "Stopband-expanded low-pass filters using microstrip coupled-line hairpin units," IEEE Microwave and Wireless Components Letters, vol. 18, no. 8, pp. 506-508, 2008.

[8] R. Gómez-García, M.-Á. Sánchez-Soriano, M. SánchezRenedo, G. Torregrosa-Penalva, and E. Bronchalo, "Low-pass and bandpass filters with ultra-broad stopband bandwidth based on directional couplers," IEEE Transactions on Microwave Theory and Techniques, vol. 61, no. 12, pp. 4365-4375, 2013.

[9] D. Kaddour, E. Pistono, J. Duchamp et al., "A compact and selective low-pass filter with reduced spurious responses, based on CPW tapered periodic structures," IEEE Transactions on Microwave Theory and Techniques, vol. 54, no. 6, pp. 2367-2375, 2006.

[10] J.-W. Sheen, "A compact semi-lumped low-pass filter for harmonics and spurious suppression," IEEE Microwave and Wireless Components Letters, vol. 10, no. 3, pp. 92-93, 2000.

[11] V. K. Velidi and S. Sanyal, "Sharp roll-off lowpass filter with wide stopband using stub-loaded coupled-line hairpin unit," IEEE Microwave and Wireless Components Letters, vol. 21, no. 6, pp. 301-303, 2011. 

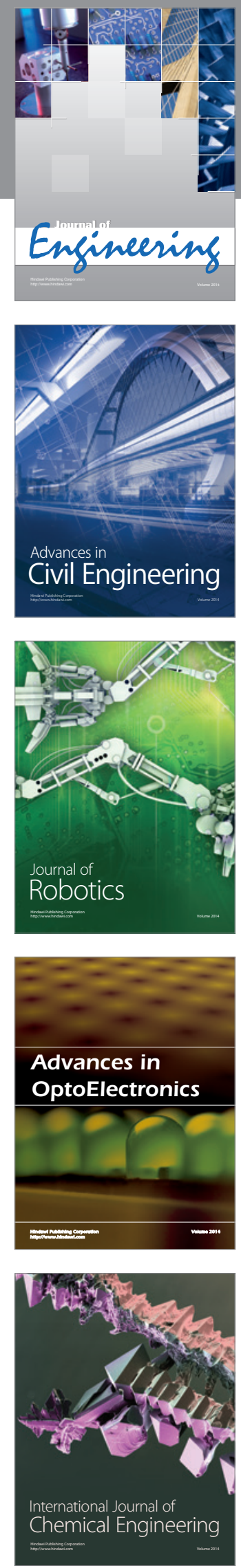

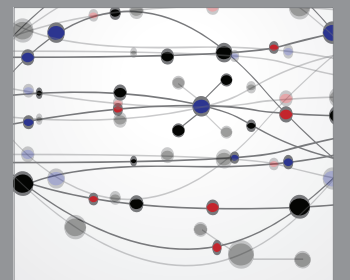

The Scientific World Journal
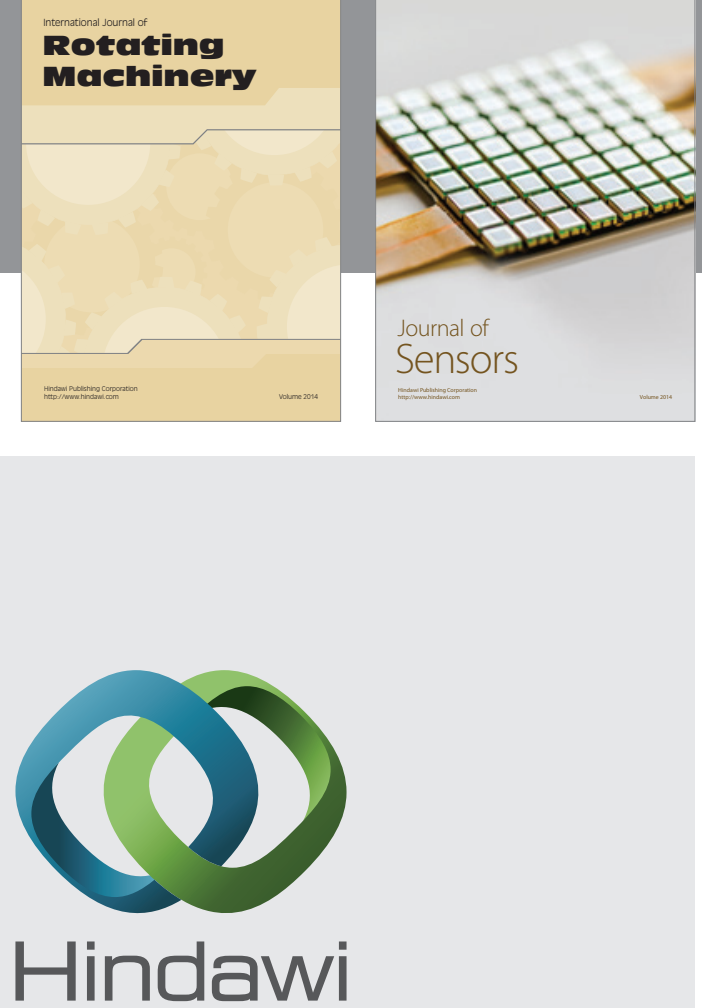

Submit your manuscripts at http://www.hindawi.com
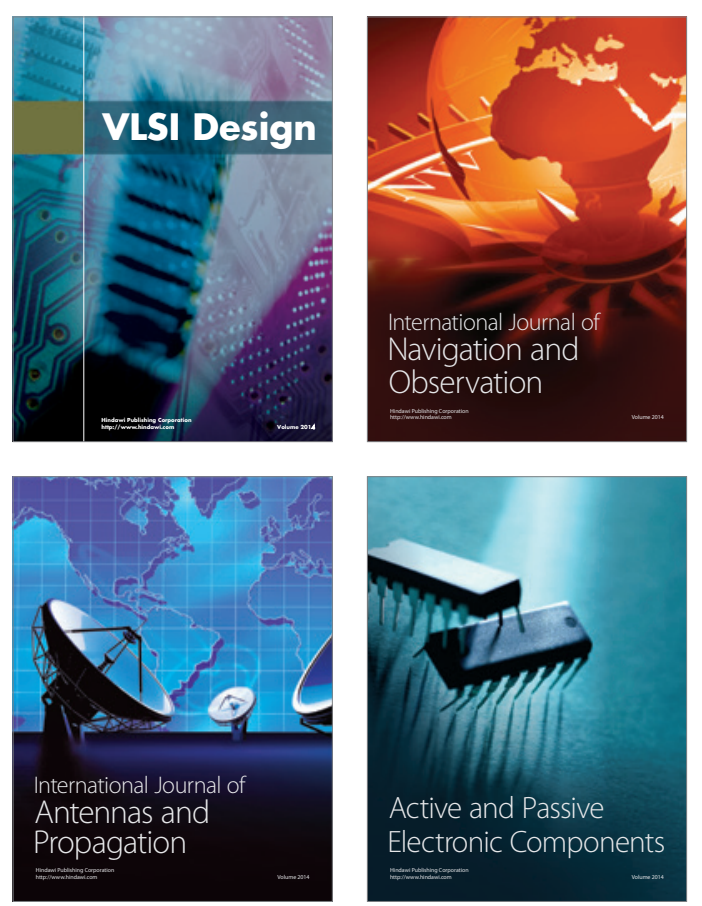
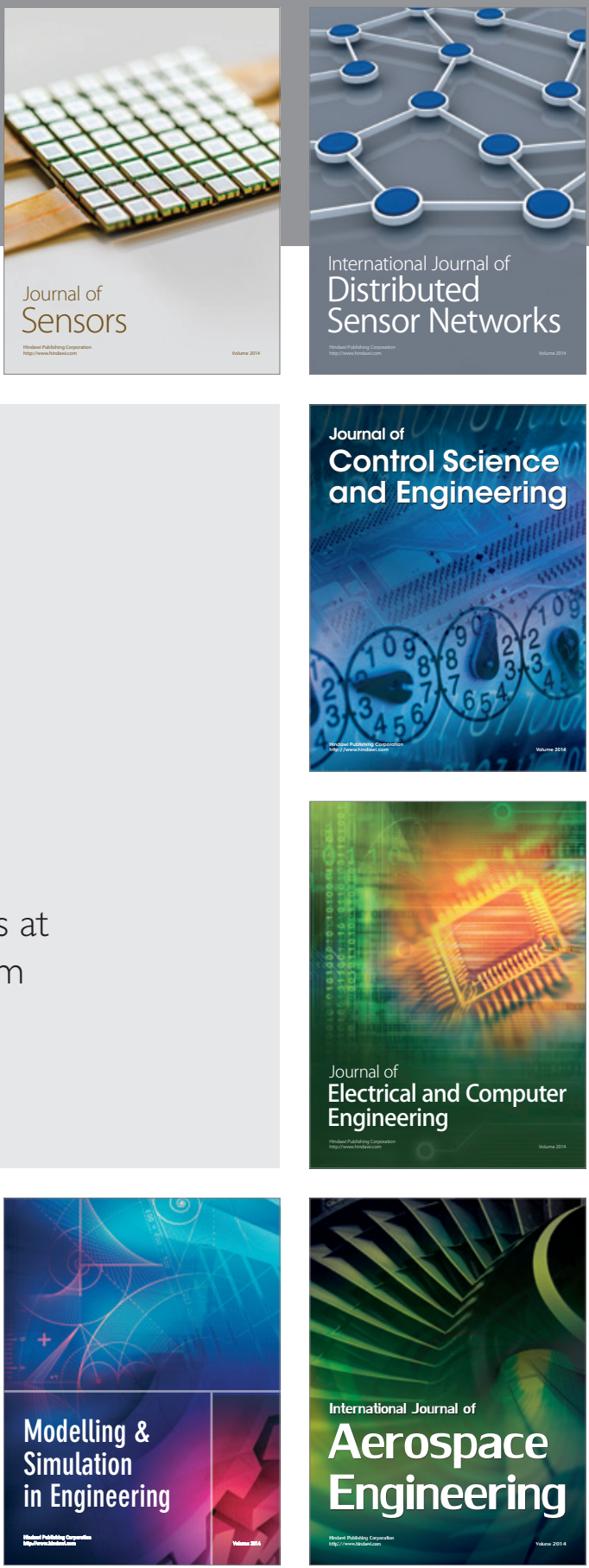

Journal of

Control Science

and Engineering
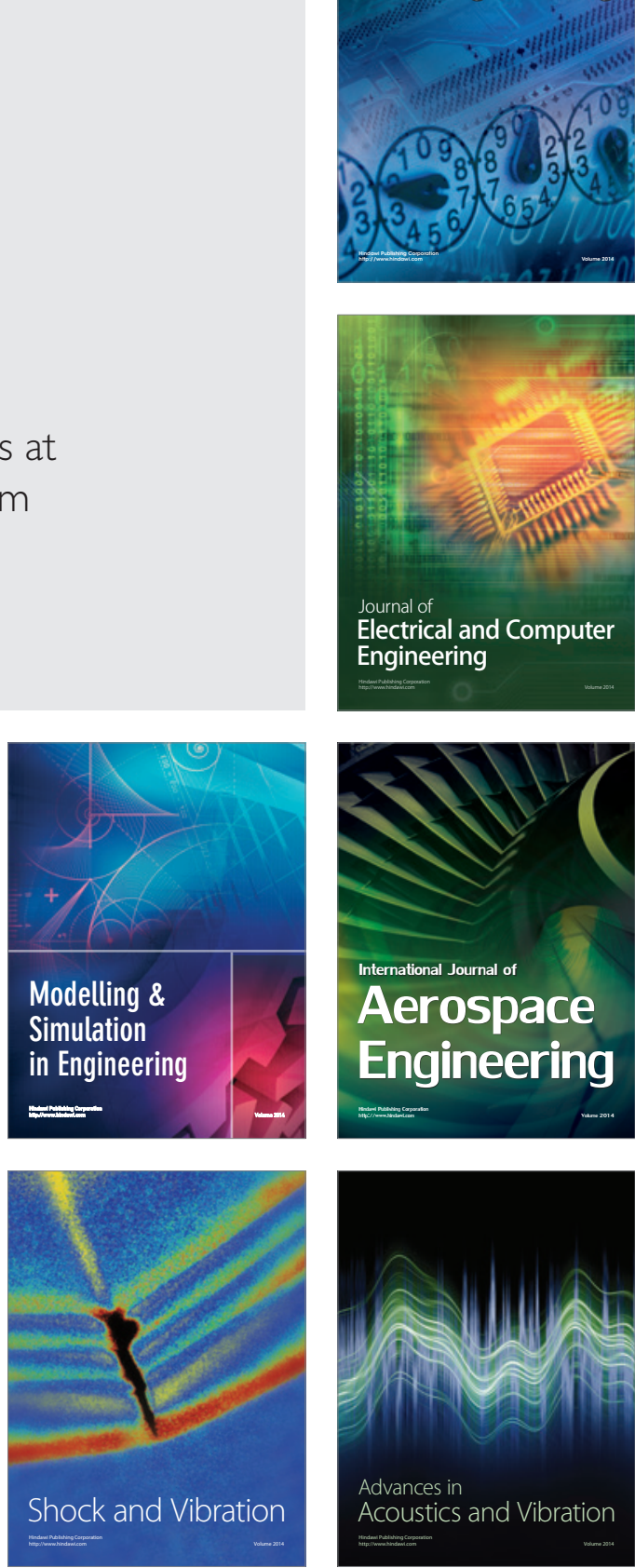